Journal of Computer Science 7 (10): 1490-1496, 2011

ISSN 1549-3636

(C) 2011 Science Publications

\title{
Evaluation of Multiple Input Multiple Output (MIMO) System Capacity with Spatial Correlations
}

\author{
${ }^{1}$ T.Gunasekaran and ${ }^{2}$ K.R.Shankar Kumar \\ ${ }^{1}$ Department of Electronics and Communication Engineering, \\ Vivekanandha College of Technology for Women, Tiruchengode, \\ ${ }^{2}$ Sri Ramakrishna Engineering College, Coimbatore, \\ Anna University of Technology, Tamil Nadu, India
}

\begin{abstract}
Problem statement: Today's wireless technologies just won't get us to the hyper connectivity of uninterrupted access from any mobile device with unlimited bandwidth at real-time speeds. This is due to the low data throughput and scarcity in spectrum. Approach: Under the condition of uncorrelated antenna elements, capacity of the Multiple Input Multiple Output (MIMO) system can be increased linearly with the number of antennas. But in practice correlation among the antenna elements exists and it can reduce the channel capacity. The effect of correlation can be reduced by using spatial diversity techniques. Results: The capacity of various MIMO configurations were analyzed and simulated. The effect of capacity of MIMO system due to correlation was presented with and without Eigen value distribution of the channel. The spatial correlation of dipole array and rectangular micro strip patch arrays were simulated with respect to various element spacing. Conclusion/Recommendations: The simulation results showed that, the capacity of MIMO system without spatial correlation was higher than with correlation effect. By comparing, the rectangular micro strip patch array has low correlation than dipole array and provides high capacity which can be utilized for high data rate applications.
\end{abstract}

Key words: Channel capacity, eigen distribution, rectangular micro strip patch arrays, spatial correlation, wireless communication, Multiple Input Multiple Output (MIMO) system, SISO system

\section{INTRODUCTION}

Wireless communication is rapidly becoming the most popular solution to deliver voice and data services due to flexibility and mobility that can be offered at moderate infrastructure costs. Today's wireless technologies just won't get us to the hyper-connectivity of uninterrupted access from any mobile device with unlimited bandwidth at real-time speeds. This is due to the low data throughput and scarcity in spectrum. In future, getting the most out of the sweet spot in spectrum where bandwidth-hungry applications like video and IPTV are transmitted will be critical to continuous mobile users. Hence Wireless technology must deliver ever increasing performance while being challenged to manage limited available spectrum. Ideally, a technique that can more efficiently deliver faster and higher quality signals without the use of additional bandwidth is required.

The Multiple Input Multiple Output (MIMO) approach is a compelling one that addresses the wireless communication challenges such as signal fading, increasing interference, and limited spectrum. MIMO multiplies data throughput, and provides for a simultaneous increase in range and reliability, all without consuming extra bandwidth. Under the condition of uncorrelated antenna elements, capacity of the MIMO system can be increased linearly with the number of antennas (Foschini and Gans, 1998).

But in practice correlation among the antenna elements exists and it can reduce the channel capacity. Hence the primary aim of any MIMO system is to reduce correlation between received signals.

To reduce the correlation the following techniques can be adopted: Employing diversity techniques and changing the antenna configuration. The effect of correlation can be reduced by increasing the spacing between the antennas. But in handheld devices, the space is limited. Hence something must be done to reduce correlation and increase the channel capacity.

MIMO systems-A review: MIMO is a multidimensional approach that transmits and receives two

Corresponding Author: T.Gunasekaran, Department of Electronics and Communication Engineering,

Vivekanandha College of Technology for Women, Tiruchengode. 
or more unique data streams through one channel whereby the system delivers two or more times the data rate per channel. MIMO is the only technique that increases capacity by delivering two or more times the data rate of a system per channel or per $\mathrm{MHz}$ of spectrum (Shiu et al., 2004).

SISO, SIMO and MISO are degenerate cases of MIMO. Multiple-Input and Single-Output (MISO) is a degenerate case when the receiver has a single antenna. Single-Input and Multiple-Output (SIMO) is a degenerate case when the transmitter has a single antenna. Single-Input Single-Output (SISO) is a system where both the transmitter and the receiver have a single antenna.

SISO system: This is the conventional system that is used everywhere. This is shown in the Fig 1. SISO is relatively simple and cheap to implement and it has been used age long since the birth of radio technology.

If bandwidth is $\mathrm{B}$, transmitted power is $\mathrm{P}$ and received Signal to Noise Ratio is SNR then the channel capacity (C) in bits $\sec ^{-1}$ is given by Shannon's theorem.

$\mathrm{C}=\mathrm{B} \cdot \log _{2}(1+\mathrm{SNR})$

SIMO system: To improve performance of a SISO system, a multiple antenna technique has been developed. A system which uses a single antenna at the transmitter and multiple antennas at the receiver is named Single Input Multiple Output (SIMO). This is shown in the Fig 2.

The channel capacity of a SIMO system is given by.

$\mathrm{C}=\mathrm{B} \cdot \log _{2}(1+\mathrm{N} \cdot \mathrm{SNR})$

where, $\mathrm{N}$ is the number of antennas at the receiver.

MISO system: A system which uses multiple antennas at the transmitter and a single antenna at the receiver is named as Multiple Input Single Output (MISO). This is shown in the Fig 3.

The channel capacity of a MISO system is given by.

$\mathrm{C}=\mathrm{B} \cdot \log _{2}(1+\mathrm{M} \cdot \mathrm{SNR})$

where, $\mathrm{M}$ is the number of antennas at the transmitter.

In MIMO systems, a transmitter sends multiple streams by multiple transmit antennas. The transmit streams go through a matrix channel which consists of multiple paths between the transmitter and the receiver. Then, the receiver gets the received signal vectors by the multiple receive antennas and decodes the received signal vectors into the original information. Fig 4. shows the model of a MIMO system.

They transmit independent data (say $\mathrm{x} 1, \mathrm{x} 2 \ldots, \mathrm{xM}$ ) on different transmit antennas simultaneously and in the same frequency band. Representing the signal received by each antenna as rj we have.

$\mathrm{r}_{1}=\mathrm{h}_{11} \mathrm{x}_{1}+\mathrm{h}_{12} \mathrm{x}_{2}+\ldots \ldots . .+\mathrm{h}_{1 \mathrm{~N}} \mathrm{x}_{\mathrm{N}}$

$\mathrm{r}_{2}=\mathrm{h}_{21} \mathrm{x}_{1}+\mathrm{h}_{22} \mathrm{x}_{2}+\ldots \ldots . .+\mathrm{h}_{2 \mathrm{~N}} \mathrm{x}_{\mathrm{N}}$

$\ldots . . \mathrm{r}_{\mathrm{N}}=\mathrm{h}_{\mathrm{N} 1} \mathrm{x}_{1}+\mathrm{h}_{\mathrm{N} 2} \mathrm{x}_{2}+\ldots \ldots .+\mathrm{h}_{\mathrm{NN}} \mathrm{x}_{\mathrm{N}}$

The received and transmitted signals are related as.

$\mathrm{r}=\mathrm{Hx}+\mathrm{n}$
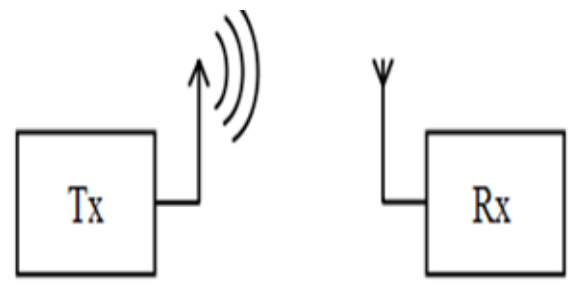

Fig. 1: SISO system
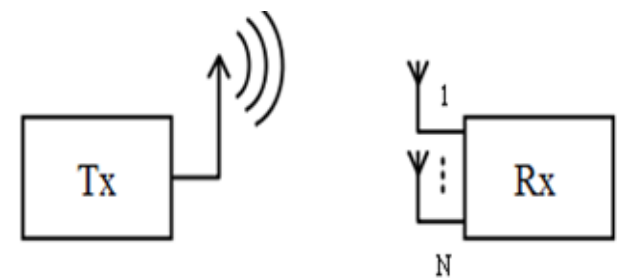

Fig. 2: SIMO system
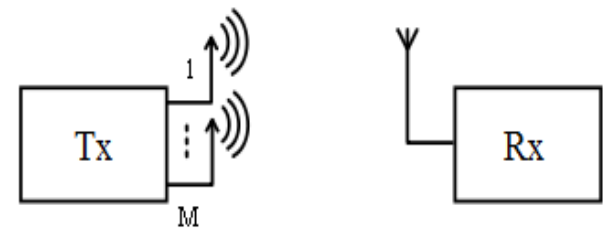

Fig. 3: MISO system
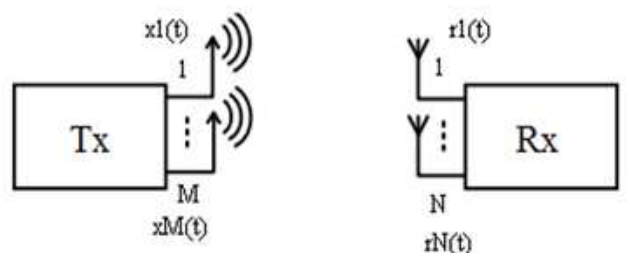

Fig. 4: MIMO system 
where, $\mathrm{n}$ is the spatial additive white Gaussian noise vector.

Working of MIMO system: MIMO takes advantage of "multi-path".

MIMO uses multiple antennas to send parallel signals from the transmitter. In an urban environment, these signals will encounter interference as they hit on trees, buildings, etc. These signals will bounce off the object and continue on their way to the receiver but in different directions.

Multi-path occurs when the different signals arrive at the receiver at various times. With MIMO, the receiving end uses an algorithm or special signal processing to sort out the received signals to produce one signal that has the originally transmitted data.

Categories of MIMO: MIMO systems can be operated in two modes depending upon whether the user requires improve in SNR or increase in capacity. This involves Spatial Diversity and Spatial Multiplexing.

Spatial Diversity- Same data is coded and transmitted through different antennas, which effectively doubles the power in the channel. This improves Signal to Noise Ratio (SNR).

Spatial Multiplexing- A high rate signal is split into multiple lower rate streams and each stream is transmitted from a different transmit antenna in the same frequency channel. If these signals arrive at the receiver antenna array with sufficiently different spatial signatures, the receiver can separate these streams, creating parallel channels for free. Spatial multiplexing is very powerful technique for increasing channel capacity (Lozano et al., 2010).

Capacity of MIMO systems: In a MIMO system with small number of antennas, the capacity grows linearly with the number of antenna elements, rather than logarithmically. This is true in a wireless link with mtransmit and n-receive channels, provided the $\mathrm{n}$ by $\mathrm{m}$ channel matrix has ideal independently fading elements.

Unfortunately, several practical issues pose problems for achieving the predicted high capacity. To create uncorrelated channels in a MIMO system, the spacing between antenna elements must be in the order of a few wavelengths. This is because MIMO uses space diversity to create uncorrelated channels. The farther the antennas are from each other, the less correlation the multi-path would experience.

The instantaneous channel capacity of a MIMO channel $\mathrm{H}$ can be written as.

$$
\mathrm{C}=\log _{2}\left(\operatorname{det}\left[\mathrm{I}+\frac{\zeta}{\mathrm{N}} \mathrm{HH}^{\dagger}\right]\right)
$$

where $\zeta$ is the average SNR at the receiver, $\mathrm{N}=$ $\min \left(\mathrm{N}_{\mathrm{t}}, \mathrm{N}_{\mathrm{r}}\right)$ and $\dagger$ denotes Hermitian conjugate. Note that the channel matrix $\mathrm{H}$ is $\mathrm{N}_{\mathrm{t}}, \mathrm{N}_{\mathrm{r}}$, where $\mathrm{N}_{\mathrm{t}}, \mathrm{N}_{\mathrm{r}}$, denotes the number of antenna elements at the receiver and transmitter, respectively. The element $\mathrm{H}_{\mathrm{ij}}$ represents the complex path gain from transmitter $\mathrm{j}$ to $\mathrm{i}$ receiver.

MIMO channel from multi users, which are moving at vehicular speed, to the Base-Station is timevarying. For time-varying channels, Sphere Decoding was introduced to perform ML decoding. The computational complexity of this method is quite high (Sayadi et al., 2009). The simplest approach for MIMO channel modeling is to assume an i.i.d. complex Gaussian fading MIMO channel that provides both large capacity and diversity. Actually correlations at both Rx (receiver) and Tx (transmitter) are very likely to exist because of limited angular spread and dominant directions of arrival or departure. To account for this it is necessary to include the correlation of the channel matrix elements in a proper way. One such model widely used is the Kronecker model. Hence this model is assumed for the channel modeling and analysis (Chen et al., 2010).

Spatial correlation: Spatial correlation is one of the substantial factors in Multiple Input Multiple Output (MIMO) wireless communication systems. Thus, correlation between antennas could be crucial for the performance of the system. The spatial correlation must be therefore taken into account in system simulations.

In particular, the advantage of the rich scattering radio environment causes MIMO systems to obtain higher benefits due to low correlated channel. In the case of a scattering environment, separating the elements in the receive and transmit arrays by at least $0.4 \lambda$ would ensure less correlation. While this does not present a problem in a base station, accommodating multipleantenna arrays in a small portable device with such a separation between antenna elements might not be possible. Spatial correlation is a key factor (Lozano et al., 2010) that affects the capacity of MIMO systems. The smaller the spatial correlation better is the output performance.

\section{MATERIALS AND METHODS}

In this study, the capacity of a MIMO system which uses a uniform rectangular patch microstrip array is evaluated for various values of SNR, after introducing the spatial correlation matrix. As the spatial correlation matrix entries are calculated by taking azimuth spread and elevation spread into account, the channel model is more realistic and gives the influence of the antenna array on the capacity. 
Evaluating spatial correlation: The spatial correlation evaluation is useful to calculate MIMO capacity and to obtain channel state information (Yong and Thompson, 2003). Here, the spatial correlation between two antenna elements of an array is derived. In general, the spatial correlation between antenna 1 and antenna 2 when only azimuth power spectrum is considered is given by:

$\rho=\frac{\int_{\phi_{0}-\Delta_{\phi}}^{\phi_{0}+\Delta_{\phi}} \mathrm{E}_{1}(\phi) \mathrm{E}_{2}^{*}(\phi) \mathrm{p}(\phi) \mathrm{d} \phi}{\sqrt{\mathrm{P}_{1} \mathrm{P}_{2}}}$

The spatial correlation between antenna 1 and antenna 2 when both azimuth and elevation planes are considered is given by:

$\rho=\frac{\int_{\phi_{0}-\Delta_{\phi}}^{\phi_{0}+\Delta_{\phi}} \int_{\theta_{0}-\Delta_{\theta}}^{\theta_{0}+\Delta_{\theta}} \mathrm{E}_{1}(\theta, \phi) \mathrm{E}_{2}{ }^{*}(\theta, \phi) \mathrm{p}(\theta, \phi) \sin \theta \mathrm{d} \theta \mathrm{d} \phi}{\sqrt{\mathrm{P}_{1} \mathrm{P}_{2}}}$

where, $E_{1}(\theta, \phi)$ and $E_{2}(\theta, \phi)$ are the radiation patterns of antennas 1-2 respectively. $\mathrm{P}_{1}, \mathrm{P}_{2}$ are the mean received powers and $\mathrm{p}(\theta, \phi)$ is the joint probability density function of the angle of arrivals. $2 \Delta_{\theta}$ and $2 \Delta_{\phi}$ are the elevation and azimuth spreads respectively. The expression for mean power is given by.

$\mathrm{P}_{\mathrm{i}}=\int_{\phi_{0}-\Delta_{\phi}}^{\phi_{0}+\Delta_{\phi}} \int_{\theta_{0}-\Delta_{\theta}}^{\theta_{0}+\Delta_{\theta}}\left|\mathrm{E}_{\mathrm{i}}(\theta, \phi)\right|^{2} \mathrm{p}(\theta, \phi) \sin \theta \mathrm{d} \theta \mathrm{d} \phi$

The normalized radiation pattern of a rectangular microstrip (Balanis, 1997) is shown in eq. (10)

$$
\mathrm{E}(\theta, \phi)=\left\{\sin \theta \frac{\sin \left(\frac{\mathrm{k}_{0} \mathrm{~W}}{2} \cos \theta\right)}{\cos \theta}\right\} \cos \left(\frac{\mathrm{k}_{0} \mathrm{~L}_{\mathrm{e}}}{2} \sin \theta \sin \phi\right)
$$

Using (10) in (8) we get equation (A1).

The integral in (A1) can be evaluated if we can identify:

$\mathrm{Z}_{\mathrm{x}}=2 \pi \mathrm{d}_{\mathrm{x}}(\mathrm{n}-\mathrm{m}) / \lambda$,

$\mathrm{Z}_{\mathrm{y}}=2 \pi \mathrm{d}_{\mathrm{y}}(\mathrm{p}-\mathrm{q}) / \lambda$

Let $Z=\sqrt{Z_{x}^{2}+Z_{y}^{2}}$ and $\gamma=\tan ^{-1}\left(Z_{x} / Z_{y}\right)$, equation (A1) can be written as (A2).
Let:

$$
\begin{aligned}
& \mathrm{a}=\phi_{0}+\gamma-\Delta_{\phi}, \\
& \mathrm{b}=\phi_{0}+\gamma+\Delta_{\phi}, \text { and } \mathrm{d}=\theta_{0}+\Delta_{\theta} \\
& \mathrm{c}=\theta_{0}-\Delta_{\theta}
\end{aligned}
$$

The Eq. (A3-A4) gives the values for spatial correlation and is evaluated numerically.

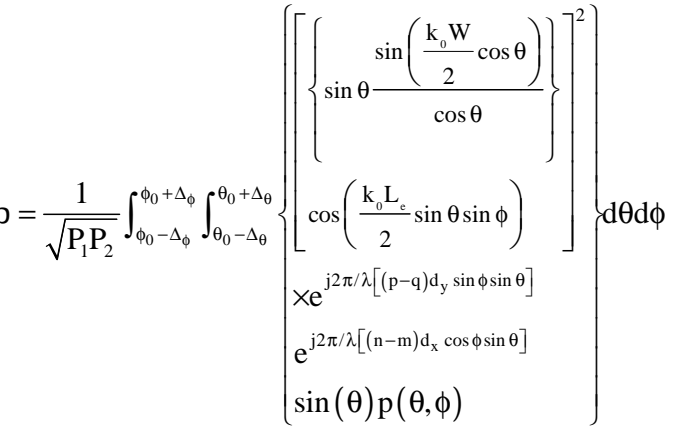

$$
\begin{aligned}
& \rho=\mathrm{k} \int_{\phi_{0}-\Delta \phi}^{\phi_{0}+\Delta_{\phi}} \int_{\theta_{0}-\Delta_{\theta}}^{\theta_{0}+\Delta_{\theta}} \mathrm{e}^{\mathrm{j} Z \sin \theta \sin (\gamma+\phi)}\left[\begin{array}{l}
\left\{\frac{\sin \left(\frac{\mathrm{k}_{0} \mathrm{~W}}{2} \cos \theta\right)}{\cos \theta}\right\} \\
\cos \left(\frac{\mathrm{k}_{0} \mathrm{~L}_{\mathrm{c}}}{2} \sin \theta \sin \phi\right)
\end{array}\right]^{2} \\
& \sin ^{3}(\theta) d \theta d \phi \\
& \operatorname{Im}(\rho)=k \int_{a}^{b} \int_{c}^{d} \sin (Z \sin \theta \sin \psi)\left[\begin{array}{l}
\left\{\frac{\sin \left(\frac{k_{0} W}{2} \cos \theta\right)}{\cos \theta}\right\} \\
\cos \left(\frac{k_{0} L_{c}}{2} \sin \theta \sin \phi\right)
\end{array}\right]^{2} \\
& \sin ^{3}(\theta) d \theta d \psi \\
& \operatorname{Re}(\rho)=\mathrm{k} \int_{\mathrm{a}}^{\mathrm{b}} \int_{\mathrm{c}}^{\mathrm{d}} \cos (\mathrm{Z} \sin \theta \sin \psi)\left[\begin{array}{c}
\left.\left\{\frac{\sin \left(\frac{\mathrm{k}_{0} \mathrm{~W}}{2} \cos \theta\right)}{\cos \theta}\right\}\right]^{2} \\
\cos \left(\frac{\mathrm{k}_{0} \mathrm{~L}_{\mathrm{c}}}{2} \sin \theta \sin \phi\right) \\
\sin ^{3}(\theta) \mathrm{d} \theta \mathrm{d} \psi
\end{array}\right.
\end{aligned}
$$

where $\mathrm{k}=\frac{1}{\sqrt{\mathrm{P}_{1} \mathrm{P}_{2}}}$ and is evaluated numerically.

The joint spatial power spectrum $p(\theta, \phi)$ can be decomposed as $p(\theta) p(\phi)$, by assuming that the angles of arrivals in azimuth and elevation planes are independent (Yong and Thompson, 2003). 


\section{RESULTS AND DISCUSSION}

In a MIMO system, the capacity grows linearly with the number of antenna elements, rather than logarithmically as shown in the Fig. 5.

The effect of capacity due to correlation can be understood from the Eigen value distribution. Here a $4 \times 4$ MIMO system has been considered to show the effect on capacity. Without considering the correlation effect, the Eigen values of the channel matrix are plotted in the Fig. 6a. As it can be observed that, a $4 \times 4$ MIMO system without correlation has its Eigen values greater than zero. Since the capacity is mean product of the Eigen values, the capacity of this system without correlation will be high. Now for the same $4 \times 4$ MIMO system, the Eigen value distribution with correlation has been plotted in the Fig. 6b. As it can be observed that, a $4 \times 4$ MIMO system with correlation has some of its Eigen values nearer to zero. This makes capacity to be low which is the main limitation in any MIMO system.

In Fig. 7.1-7.2, considering only the azimuth plane, the SC of microstrip array is compared with the SC of dipole array. The mean angle of arrival in the azimuth plane is kept at $45^{\circ}$ for the results of Fig. 7.1-7.2.

It can be noted that the SC of microstrip is lower than dipole from Fig. (7.1-7.2-7.3). The spatial correlation has also been evaluated for the various values of AS and ES as in the Fig. 8. It is very clear that the ES does not affect SC as much as the AS does, if AS is high. It can also be noted that the SC is relatively larger when AS is small and vice versa.

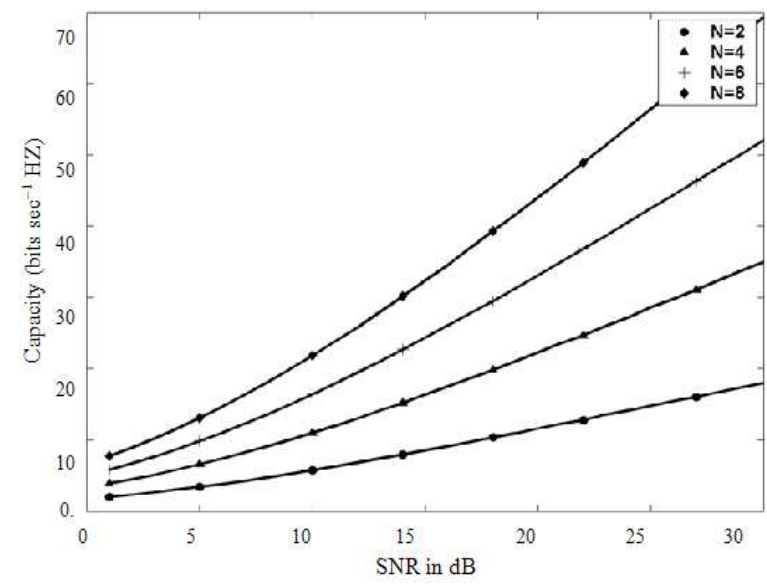

Fig. 5: Capacity of a MIMO system for various values of $\mathrm{N}$

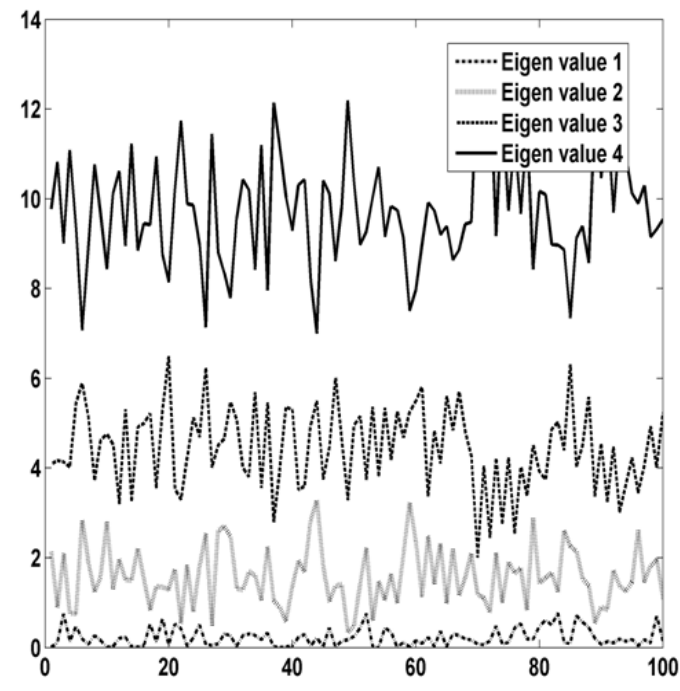

(a)

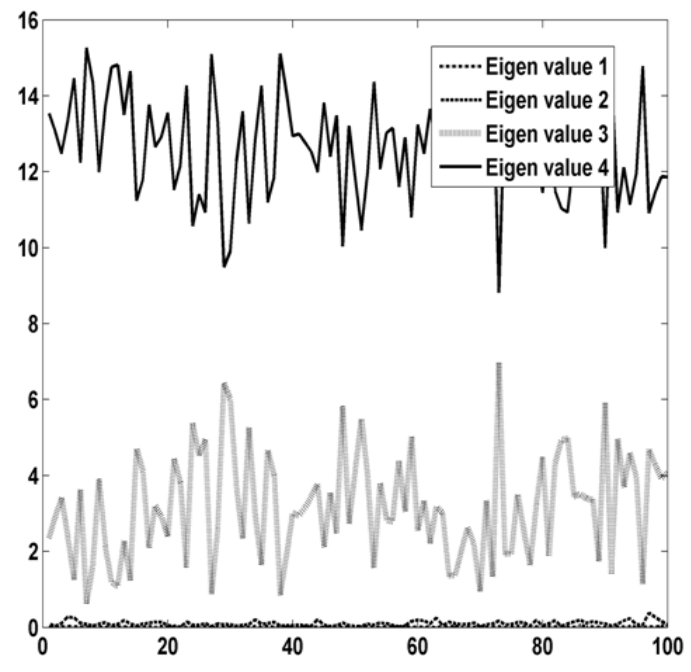

(b)

Fig. 6: a-b Eigen value distribution of a $4 \times 4$ MIMO system without and with correlation respectively 
Also considering both AS and ES, the SC of microstrip array is compared with the SC of dipole array in Fig 7.3.

The mean angles of arrival in the azimuth and elevation planes are kept at $45-90^{\circ}$ for the results of Figs. 7.3- 7.4.

The capacity of a $4 \times 4$ MIMO system was evaluated with the following settings: the antenna spacing at the transmitter is $4 \lambda$ and at the receiver is $1 \lambda$, the mean angles of arrival in the elevation and azimuth planes are $90-45^{\circ}$ with $\mathrm{AS}=\mathrm{ES}=80^{\circ}$. The capacity of the independently faded, identically distributed (i.i.d.) Gaussian channel, dipole arrays and microstrip arrays as a function of SNR, were shown in Fig. 9.

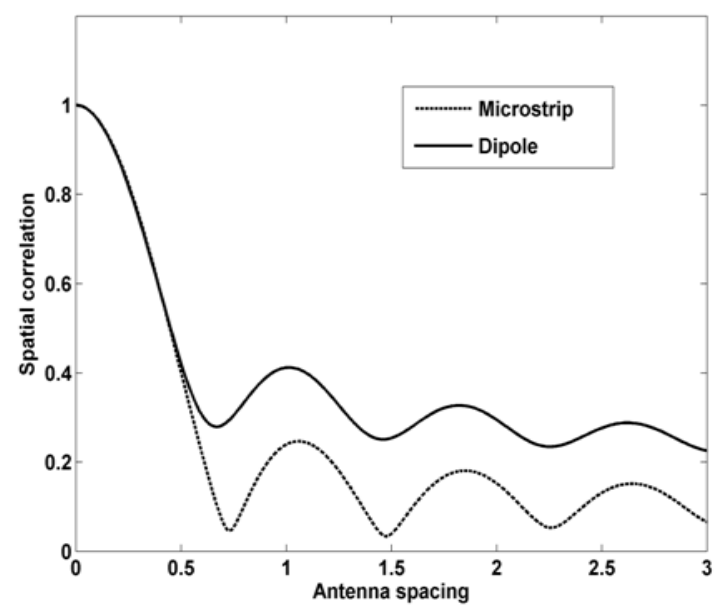

(a)

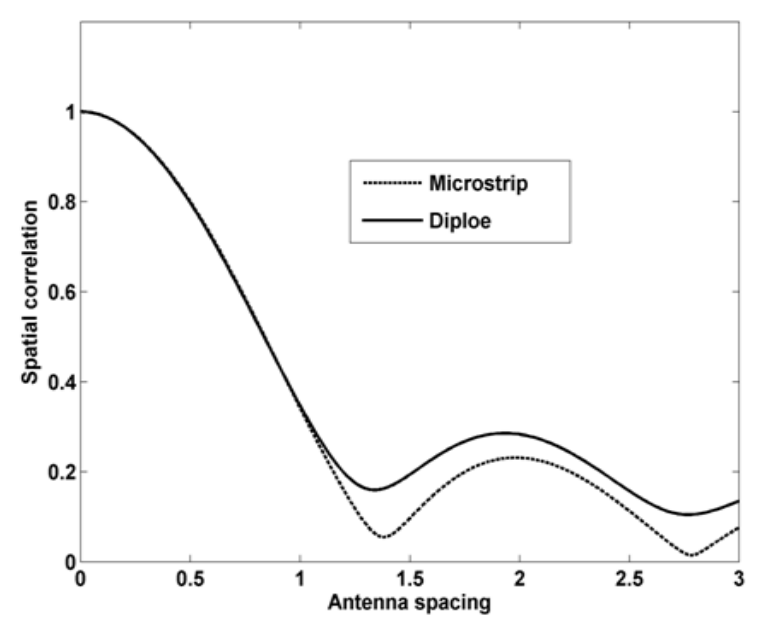

(b)

Fig. 7.1 and 7.2 Comparison of spatial correlation between micro strip and Dipole antennas for AS = 120 and 60 respectively

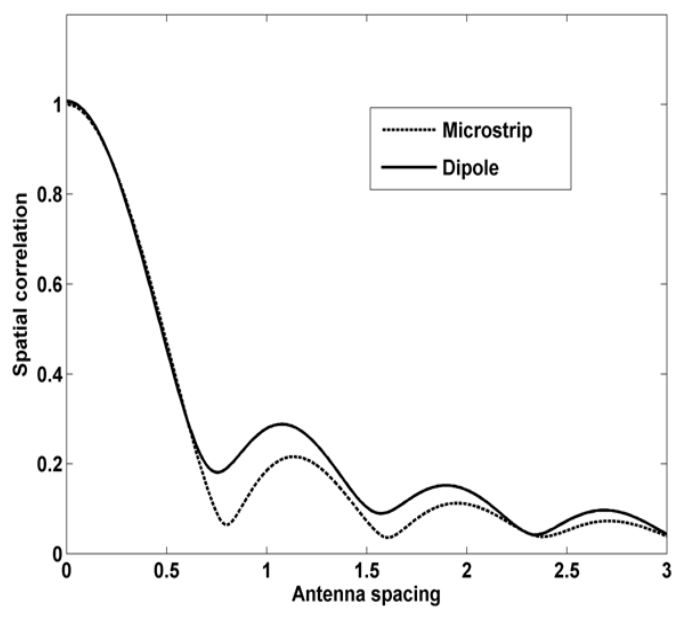

Fig. 7.3: SC Comparison between microstrip and dipole array for $\mathrm{AS}=\mathrm{ES}=80$

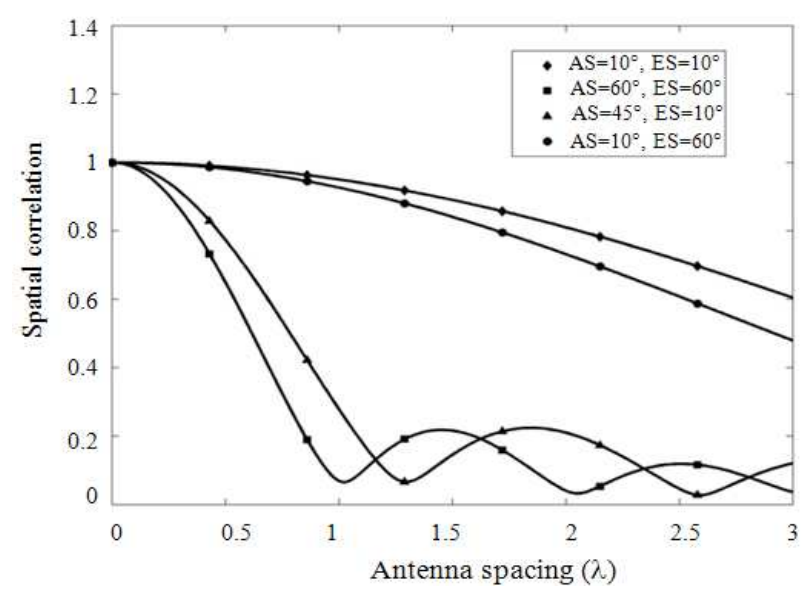

Fig. 8: SC versus antenna spacing in wavelengths

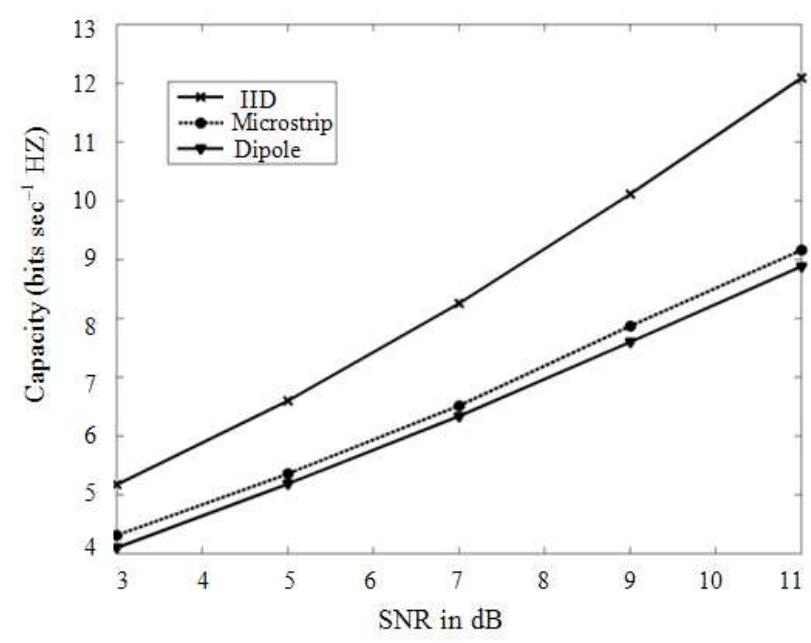

Fig. 9: Mean capacity as a function of SNR in $\mathrm{dB}$ 1495 


\section{CONCLUSION}

We showed that the channel capacity of the MIMO system have affected by the spatial correlation. It has also been verified that the capacity of the MIMO antennas without correlation is greater than that of with correlation. Simulation results proved that the rectangular patch microstrip arrays have less spatial correlation than dipole arrays and hence it can be used widely in any MIMO system. It has also been proved that the capacity of the array of rectangular patch microstrip antennas is greater than that of the dipole antenna array. In future, more work on the antenna design is needed to find suitable positions of the feeds, appropriate dimensions, and a matching network that minimizes the losses in the antenna.

\section{REFERENCES}

Foschini, G. J. and M. J. Gans, 1998. On limits of wireless communications in a fading environment when using multiple antennas. Wireless Personal Commun., 6: 311-355. DOI: 10.1023/A:1008889222784

Yong, S.K. and J.S. Thompson, 2003. A threedimensional spatial fading correlation model for uniform rectangular arrays. IEEE Antennas Wireless Propag. Lett., 2: 182-185. DOI: 10.1109/LAWP.2003.819666
Yong, S.K. and J.S. Thompson, 2003. The Impact of angle of arrival distribution on the performance analysis of different arrays architectures. In Proc, of 12th IEE International Conference on Antennas and Propagation, Mar. 2003, UK, pp: 840-843, DOI: $10.1049 / \mathrm{cp}: 20030207$

Yong, D., G.J. Foschini, M.J. Gans, and J.M. Kahn, 2000. Fading correlation and its effect on the capacity of multi-element antenna systems. IEEE Trans. Commun., 48: 502-513. DOI: 10.1.1.116.5688

Balanis, C.A., 1997. Antenna Theory: Analysis and Design, 2nd Edn., John Wiley, New York, pp: 941.

Lozano, A. and N. Jindal, 2010. Transmit diversity vs. spatial multiplexing in modern MIMO systems. IEEE Trans. Wireless Commun., 9: 186-197. DOI: 10.1109/TWC.2010.01.081381

Sayadi, F., M. Ismail, N. Misran, K.M. Jumari and M. Abdullah, 2009. Efficient detection algorithm for a multiple-input and multiple-output multiuser multicarrier code division multiple access in timevarying channels. Am. J. Eng. Applied Sci., 2: 635-642. DOI: 10.3844/ajeassp.2009.635.642.

Chen, Y.C. and Y.T. Su, 2010. MIMO channel estimation in correlated fading environments. IEEE Trans. Wireless Commun., 9: 1108-1119. DOI: 10.1109/TWC.2010.03.081603. 